\title{
Fluctuation-dissipation ratio of the Heisenberg spin glass
}

\author{
Hikaru Kawamura \\ Department of Earth and Space Science, Faculty of Science, Osaka University, Toyonaka 560-0043, Japan
}

(June 28, 2018)

\begin{abstract}
Fluctuation-dissipation (FD) relation of the three-dimensional Heisenberg spin glass with weak random anisotropy is studied by off-equilibrium Monte Carlo simulation. Numerically determined FD ratio exhibits a "one-step-like" behavior, the effective temperature of the spin-glass state being about twice the spin-glass transition temperature, $T_{\text {eff }} \simeq 2 T_{g}$, irrespective of the bath temperature. The results are discussed in conjunction with the recent experiment by Hérisson and Ocio, and with the chirality scenario of spin-glass transition.
\end{abstract}

Off-equilibrium dynamics of spin glass (SG) has attracted much recent interest [1]. In equilibrium, there holds a relation between the response and the correlation known as the fluctuation-dissipation theorem (FDT). In off-equilibrium, relaxation of physical quantities of SG depends on its previous history. Most typically, it depends not only on the observation time $t$ but also on the waiting time $t_{w}$, i.e., exhibits aging. While in the shorttime quasi-equilibrium regime $t_{0}<<t<<t_{w}$ ( $t_{0}$ is a microscopic time scale), relaxation is still stationary and FDT holds, it becomes non-stationary and FDT is broken in the long-time aging regime $t>t_{w}$. In particular, the breaking pattern of FDT, and its possible relation to the static quantity, has been the subject of recent active studies.

A quantity playing a central role here is the so-called fluctuation-dissipation ratio (FDR) $X$, which may be defined by the relation,

$$
R\left(t_{1}, t_{2}\right)=\frac{X\left(t_{1}, t_{2}\right)}{k_{B} T} \frac{\partial C\left(t_{1}, t_{2}\right)}{\partial t_{1}}
$$

where $R\left(t_{1}, t_{2}\right)$ is a response function measured at time $t_{2}$ to an impulse field applied at time $t_{1}, C\left(t_{1}, t_{2}\right)$ is a two-time correlation function in zero field at times $t_{1}$ and $t_{2}$, and $T$ is the bath temperature. One may regard $T / X \equiv T_{\text {eff }}$ as an effective temperature. In the case FDT holds, one has $X=1$ and $T_{\text {eff }}=T$.

Via the study of certain mean-field models, Cugliandolo and Kurchan showed that, in the limit of infinite time $t_{1}, t_{2} \rightarrow \infty$, the FDR $X$ depended on the times $t_{1}$ and $t_{2}$ only through the correlation function $C\left(t_{1}, t_{2}\right)$, i.e., $X\left(t_{1}, t_{2}\right)=X\left(C\left(t_{1}, t_{2}\right)\right)$ [2]. It was further suggested that $X(C)$ could be related to an appropriate static quantity, i.e., $X(C)$ is equivalent to the $x(q)$-function describing the replica-symmetry breaking (RSB) pattern in the Parisi's scheme, which is related to the overlap distribution function via $P(q)=\frac{\mathrm{d} x(q)}{\mathrm{d} q}[2]$. This conjecture was supported both by numerical simulation [3] and analytic work [4] (see, however, also ref. [5]).

Experimental studies on the FDR of SG have been hampered for a long time by the difficulty in performing high-precision measurements of correlations (noise measurements), although some interesting preliminary results were reported [6]. Recently, however, a remarkable experiment by Hérisson and Ocio for an insulating Heisenberg SG $\mathrm{CdCr}_{1.7} \mathrm{In}_{0.3} \mathrm{~S}_{4}$ has eventually opened a door to experimental access to the FDR of SG [7]. Some comparison was already made between these expeirmental results and the numerical results obtained by offequilibrium simulation on certain SG models.

Meanwhile, it should be remembered that all numerical results of the FDR so far available are limited to the Ising-like SG models, which represent an extremely anisotropic limit [3,5,8-11]. By contrast, the SG material studied in ref. [7] was more or less Heisenberg-like. Recent experimental and numerical studies suggested that both equilibrium and off-equilibrium properties might differ significantly between the Ising and Heisneberg SGs [12-17]. Under such circumstances, in order to make a direct link between theory and experiment, it is clearly desirable to study the Heisenberg-like SG where magnetic anisotropy is weak. The purpose of the present Letter is to perform extensive off-equilibrium simulations on such a weakly anisotropic Heisenberg-like SG model, and numerically investigate its FDR. Interestingly, the calculated FDR of the three-dimensional (3D) Heisenberg SG exhibits a feature of the so-called one-step RSB.

I consider the weakly anisotropic classical Heisenberg model whose Hamiltonian is given by

$$
\mathcal{H}=-\sum_{<i j>}\left(J_{i j} \mathbf{S}_{i} \cdot \mathbf{S}_{j}+D_{i j}^{\mu \nu} S_{i}^{\mu} S_{j}^{\nu}\right)-h \sum_{i} S_{i}^{z}
$$

where $\mathbf{S}_{i}=\left(S_{i}^{x}, S_{i}^{y}, S_{i}^{z}\right)$ is a three-component unit vector at the $i$-th site, and the sum runs over all nearestneighbor pairs of a 3D simple cubic lattice with $N=L^{3}$ spins. $J_{i j}$ is the isotropic random exchange coupling taking the value $J$ or $-J$ with equal probability $( \pm J$ or binary distribution), $D_{i j}^{\mu \nu}(\mu, \nu=x, y, z)$ is the random exchange anisotropy which is assumed to be symmetric $\left(D_{i j}^{\mu \nu}=D_{i j}^{\nu \mu}\right)$ and uniformly distributed between the interval $[-D, D]$, and $h$ is a magnetic field applied along $z$-direction. Aimed at clarifying the properties in the limit of weak anisotropy, I assume here a rather weak anisotropy of $D / J=0.01$. The SG transition temperature of this model estimated from equilibrium Monte Carlo $(\mathrm{MC})$ simulation is $T_{g} / J \simeq 0.21$ [18]. 
Dynamical Monte Carlo simulation is performed according to the standard single spin-flip heat-bath method. At time zero, I quench the system from an infinite temperature to a working temperautre $T$ below $T_{g}$, and wait in zero field during the time $t_{w}$. In calculating the response, a weak DC field of intensity $h$ is applied, and subsequent growth of the magnetization per spin, $\left[<m_{z}\left(t+t_{w}\right)>\right]$, is recorded at time $t+t_{w}(<\cdots>$ and $[\cdots]$ are thermal average and average over quenched randomness). In calculating correlation, I measure the two-time correlation function of magnetization at times $t_{w}$ and $t+t_{w}, C\left(t ; t_{w}\right)=\left[<\vec{m}_{z}\left(t_{w}\right) \cdot \vec{m}_{z}\left(t+t_{w}\right)>\right]$, always staying in zero field in this case.

The integrated FD relation eq.(2) yields the zero-field cooled (ZFC) susceptibility $\chi \equiv\left[<m_{z}\left(t_{w}+t\right)>\right] / h$ as,

$$
\chi\left(t ; t_{w}\right)=\frac{1}{3 k_{B} T} \int_{C\left(t ; t_{w}\right)}^{1} X\left(C^{\prime}\right) \mathrm{d} C^{\prime},
$$

where the factor $\frac{1}{3}$ takes care of the number of components of Heisenberg spins.

The lattice size mainly studied is $L=32$ with periodic boundary conditions. I generate total of $10^{6}$ Monte Carlo steps per spin (MCS) in each run, with $t_{w}=2.5 \times 10^{4}$ or $10^{5}$ MCS. Sample average is taken over 40-160 independent bond realizations.

The calculated spin autocorrelation functions are shown in Fig.1 as a function of observation time scaled by the waiting time, $t / t_{w}$, for several temperatures below $T_{g}$. The decay of the autocorrelation at lower temperatures occurs in two steps. Near the end of the quasi-equilibrium regime, the decay curves tend to level off exhibiting a plateau-like structure, which is expected to correspond to the static Edwards-Anderson order parameter $q_{\mathrm{EA}}$ [12]. Furthermore, at lower temperatures $T / J=0.10$ and 0.05 the two curves for different $t_{w}$ cross around $t / t_{w} \sim 1$, and at $t>t_{w}$, the data for larger $t_{w}$ lie above the one for smaller $t_{w}$ (superaging). Such a superaging behavior means that the aging is apparently more enhanced than the one expected from the naive $t / t_{w}$-scaling. The observed superaging of spin correlations is similar to the one previously observed in the Heisenberg SG with the Gaussian coupling [12]. At higher temperatures $T / J=0.15$ and 0.20 , by contrast, the curve for longer $t_{w}$ lies slightly below the one for shorter $t_{w}$ (subaging), or nearly comes on top of it for longer $t$ (near full aging). To examine the robustness of the observed aging behavior against the system size and the waiting time, I show in the inset the autocorrelation for the smaller size $L=16$ for the temperatures $T / J=0.15$ and 0.05 , with $t_{w}=5 \times 10^{3}$ and $2.5 \times 10^{4}$. The superaging/subaging tendency observed for $L=32$ can also be seen here.

Hence, whether the spin correlation exhibits superaging or subaging apparently depends on the bath temperature, superaging at lower temperatures and subaging (or near full aging) at higher temperatures. In the present case, the borderline temperature is around $T / J \simeq 0.15$, corresponding to $\simeq 0.7 T_{g}$. In the recent noise experiment by Hérisson and Ocio, subaging behavior is observed at $T \simeq 0.8 T_{g}$ [7]. It might be interesting to see experimentally whether the superaging behavior as observed here ever arises at lower temperatures. In real experiment, however, care needs to be taken to an inevitable coolingtime effect which might give some bias toward apparent subaging $[19,20]$.

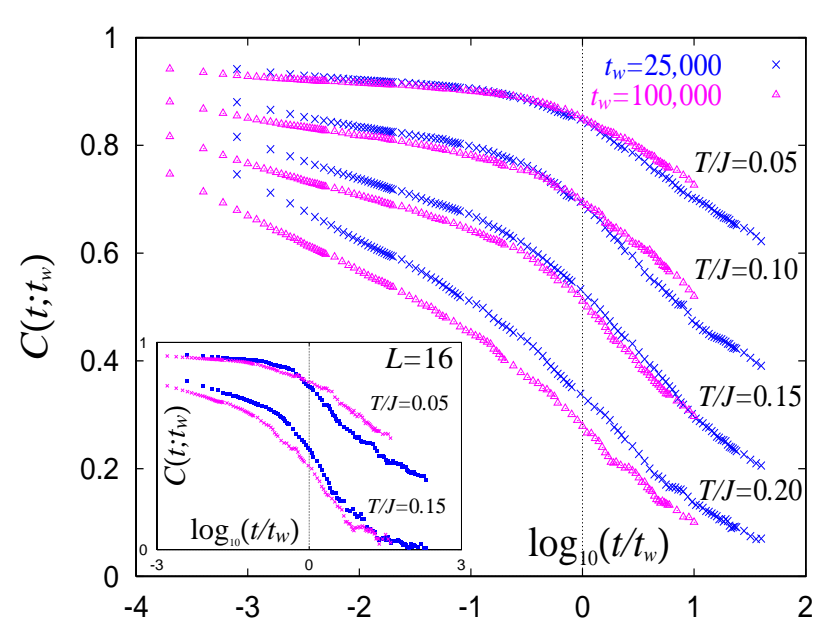

FIG. 1. Decay of the spin autocorrelation function of the weakly anisotropic $3 \mathrm{D}$ Heisenberg spin glass with $D / J=0.01$, plotted versus $\log _{10}\left(t / t_{w}\right)$ at several temperature below $T_{g}$. The lattice size is $L=32$, averaged over $80(T / J=0.20)$, $160(T / J=0.15)$ and $40(T / J=0.10)$ samples. Inset: The autocorrelation decay for the smaller system of size $L=16$ averaged over 40 samples. The waiting times are $t_{w}=5 \times 10^{3}$ (blue) and $2.5 \times 10^{4}$ (pink) here.

In the lower panel of Fig.2, I show the growth of the ZFC susceptibility $T \chi\left(t ; t_{w}\right)$ as a function of $t / t_{w}$ at several temperatures. I applied a very week field of intensity $h / J=0.01$. A word of caution is in order here. Generally, applied fields should be sufficiently weak so that one is in the linear response regime. In the previous simulations on the Ising SG, the field intensity of $h / J=0.1$ turned out to be weak enough $[3,5,9]$. In the case of Heisenberg SG, however, still weaker field appears to be necessary to guarantee the linearity of the response. This is illustrated in the upper panel of Fig.2 where I show the $t$-dependence of the ZFC susceptibility at $T / J=0.15$ for three different field intensities, $h / J=0.05,0.02$ and 0.01 . Some deviation is discernible between these curves at longer $t$, suggesting that the requirement of the linearity is rather severe in the Heisenberg case. In the following, I show the response data for the weakest field studied, $h / J=0.01$, where the linearity seems almost satisfied in the investigated time range.

Indeed, such a strong nonlinearity of the response appears to be even more enhanced in the isotropic limit $D=0$. I also made a preliminary simulation on the fully isotropic Heisenberg SG with $D=0$ at a temperature $T / J=0.15$ to study its response, with varying the field intensity $H / J=0.05,0.02,0.01$ and 0.005 . While, for a 
field of moderate intensity $H / J=0.05$, the response $\chi(t)$ of the isotropic system $D=0$ is almost common with the one of the weakly anisotropic system $D / J=0.01$, on decreasing the field intesnity, $\chi(t)$ of the isotropic system constantly gets larger at longer observation time beyond the corresponding value for $D / J=0.01$, without showing any tendency of saturation up to $H / J=0.005$. This strongly suggests that the field scale associated with the linear response is closely correlated with the strength of the mangetic anisotropy $D$, although its detailed properties are yet to be clarified.

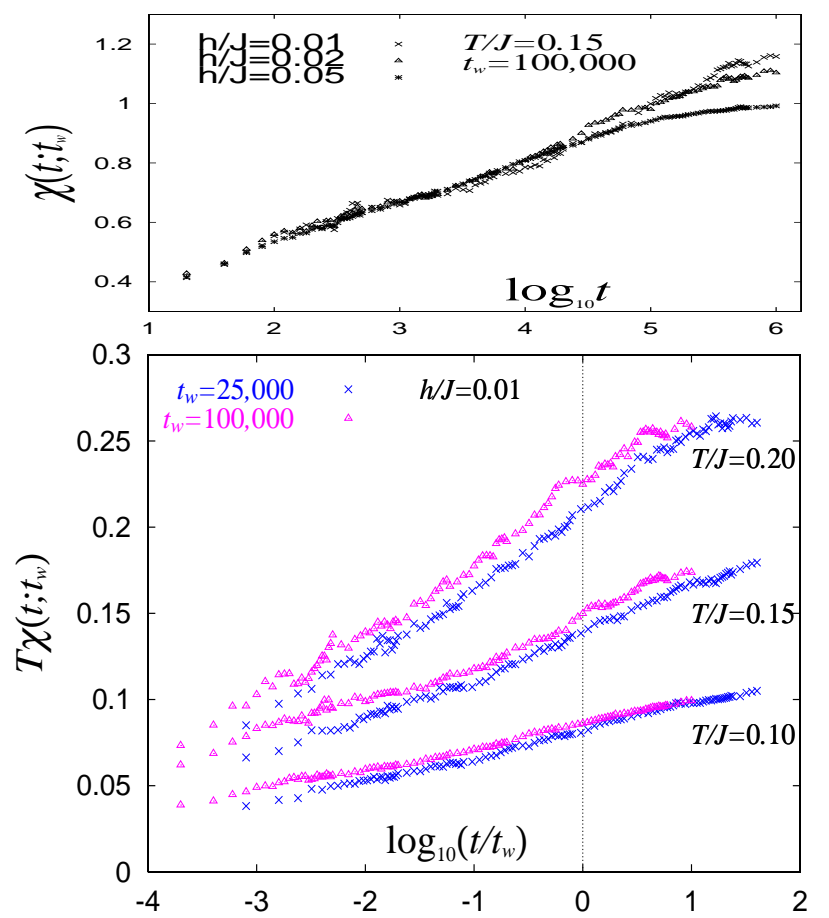

FIG. 2. Growth of the ZFC susceptibility $\chi\left(t ; t_{w}\right)$ of the weakly anisotropic 3D Heisenberg SG with $D / J=0.01$. The lattice size is $L=32$, averaged over $80(T / J=0.20), 160$ $(T / J=0.15)$ and $40(T / J=0.10)$ samples. Upper panel: $\chi\left(t ; t_{w}\right)$ plotted versus the observation time $t$ for several applied field intensities. The temperature is $T / J=0.15$, with the waiting time $t_{w}=10^{5} \mathrm{MCS}$. Lower panel: $T \chi\left(t ; t_{w}\right)$ plotted versus $\log _{10}\left(t / t_{w}\right)$ at several temperatures below $T_{g}$, with $t_{w}=2.5 \times 10^{4}$ and $10^{5} \mathrm{MCS}$.

As can be seen from the lower panel of Fig.2, the curve for longer $t_{w}$ tends to lie slightly above the one for shorter $t_{w}$ (subaging), whereas for $t>>t_{w}$ the two curves for different $t_{w}$ merge asymptoticaly (full aging). The superaging behavior, which has been seen in the correlation, is not clearly visible here in the reponse. Experimentally, response of SG usually exhibits a weak subaging $[1,7]$ or near full aging [20], which seems consistent with the present result.

In Fig.3(a), I show a parametric plot $T \chi\left(t ; t_{w}\right)$ versus $C\left(t ; t_{w}\right)$ for several temperatures. The straight line in the figure is the FDT line, $T \chi=(1-C) / 3$. In the short-time quasi-equilibrium regime, the data lie on the FDT line, and deviate from the line in the long-time aging regime. In the aging regime, the data for longer $t_{w}$ tend to lie above the data for shorter $t_{w}$, slightly, but systematically. Such a systematic drift of the data with varying $t_{w}$ is a common aspect observed both in experiment [7] and in simulations on the Ising SG [3,5,8-11].

In Fig.3(b), the data for longer $t_{w}$ are replotted in the form of $\chi\left(t ; t_{w}\right)$ versus $C\left(t ; t_{w}\right)$. One sees that the data in the aging reime at various temperatures lie on top of each other, indicating that $\chi$ is temperature independent in the aging regime, apparently satisfying Parisi-Toulouse hypothesis $[3,10]$.
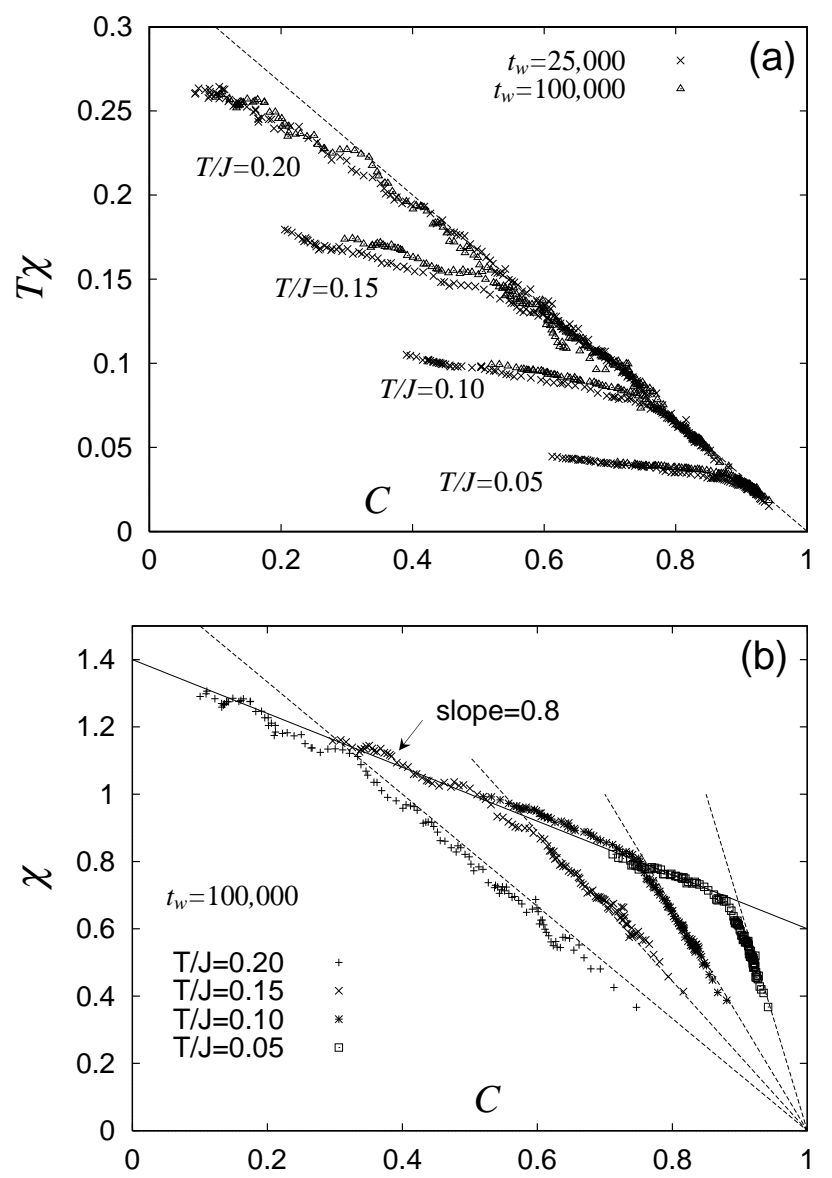

FIG. 3. $T \chi$ versus $C$ plot (a), and $\chi$ versus $C$ plot (b), of the weakly anisotropic 3D Heisenberg SG with $D / J=0.01$ at several temperatures below $T_{g}$. The applied field intensity is $h / J=0.01$. The lattice size is $L=32$, averaged over 80 $(T / J=0.20), 160(T / J=0.15)$ and $40(T / J=0.10,0.05)$ samples. In (b), $t_{w}$ is $10^{5} \mathrm{MCS}$. The broken lines represent the FDT lines. The solid line in (b) is the straight-line fit to the data in the aging regime, its slope being equal to 0.8 .

Remarkable in Fig.3 is the strikingly linearity of the plot in the aging regime, although some curvature is discernible in the part where the data begin to deviate from the FDT line. In particular, the data in the aging regime appear to come to the $C=0$ axis with a nonzero tangent. 
Such a behavior seems distinct with the behavior of the Ising SG where the data exhibit some more curvature, and appear to come to the $C=0$ axis almost horizontally, or at least be consistent with a vanishing tangent at $C=0[3,9,10]$. By making a linear fit of the data in the aging regime in Fig.3(b), I estimate the slope to be about 0.8 , which corresponds to the effective temperature $T_{\text {eff }} \simeq 0.42 J \simeq 2 T_{g}$, irrespective of the bath temperature $T\left(<T_{g} \simeq 0.21 J\right)$.

Such a one-step-like behavior of the FDR, though familiar in structural glasses, may sound surprising in SG. However, this observation finds a natural explanation in the recently developed chirality scenario of SG transition, which ascribes the true order parameter of the SG order to the chirality $[12,21]$. In this scenario, chirality exhibits a one-step-like RSB in the ordered state (chiralglass state) [13], which is manifested in the SG order in real Heisenberg-like $S G$ via the weak random magnetic anisotropy. Thus, the chirality theory predicts the occurrence of a one-step-like RSB in the SG ordered state of real Heisenberg-like SG, which, in its off-equilibrium dynamics, might well be reflected in a one-step-like behavior of the FDR. In particular, a nonzero tangent is expected at $C=0$ for the FDR, which corresponds to the delta-function peak in the static overalp distribution function $P(q)$ at $q=0[13]$. The present numerical observation seems fully consistent with such an expectation from the chirality scenario.

Now, I wish to compare the present numerical result of the FDR with the recent experiments. The one-steplike linear behavior observed in the present simulation seems at least not inconsistent with the experimental data of refs. [6,7], although the data of ref. [7] appear to exhibit some curvature showing a tendency to level off toward $C=0$. It would be interesting to experimentally explore further deep into the aging regime (smaller $C$ regime), to see whether a finite tangent persists or not at $C \rightarrow 0$. Meanwhile, the experimentally determined effective temperautre of the SG state of $\mathrm{CdCr}_{1.7} \mathrm{In}_{0.3} \mathrm{~S}_{4}$ was $T_{\text {eff }} \simeq 30 K \simeq 1.9 T_{g}\left(T_{g} \simeq 16.2 \mathrm{~K}\right)$ at the measuring temperature $T=13.3 K[7]$, which is in good agreement with the present estimate of $T_{\text {eff }}$ given above.

It should be noticed that $\mathrm{CdCr}_{1.7} \mathrm{In}_{0.3} \mathrm{~S}_{4}$, though basically a Heisenberg-like magnet, might possess a bit stronger anisotropy than the very weak anisotropy assumed here $(D / J=0.01)$. Such Ising-like tendency of $\mathrm{CdCr}_{1.7} \operatorname{In}_{0.3} \mathrm{~S}_{4}$ might be a possible cause of the curvature observed in the $\chi-C$ plot of ref. [7]. It would then be highly interesting, both experimentally and numerically, to investigate $T_{\text {eff }}$ at various bath temperatures for various systems with varying degrees of anisotropy.

In summary, I studied the fluctuation-dissipation ratio of the 3D Heisneberg SG with weak random anisotropy by off-equlibrium MC simulation. The calculated FDR exhibits a one-step-like behavior with the effective temperature of the SG state $T_{\text {eff }} \simeq 2 T_{g}$ irrespective of the bath temperature. The observed one-step-like behavior of the FDR is interpreted in terms of the recently developed chirality scenario of SG transition.

The author is thankful to M.Ocio, E.Vincent and H.Yoshino for useful discussion. The numerical calculation was performed on the Hitachi SR8000 at the supercomputer center, ISSP, University of Tokyo.

[1] For reviews, see, e.g., E. Vincent, J. Hammann, M. Ocio, J-P. Bouchaud and L.F. Cugliandolo, Sitges Conference on Glassy Systems, 1996 (Springer) cond-mat/9607224; J.-P. Bouchaud, L.F. Cugliandolo, J. Kurchan and M. Mezard, in Spin Glasses and Random Fields ed. by A.P. Young, 1997 (World Scientific), p.161; P. Nordblad and P. Svedlindh, ibid, p.1.

[2] L.F. Cugliandolo and J. Kurchan, Phys. Rev. Lett. 71, 173 (1993); J. Phys. A27, 5749 (1994).

[3] E. Marinari, G. Parisi, F. Ricci-Tersenghi and J.J. RuizLorenzo, J. Phys. A31, 2611 (1998).

[4] S. Franz, M. Mézard, G. Parisi and L. Peliti, Phys. Rev. Lett. 81, 1758 (1998).

[5] H. Yoshino, K. Hukushima and H. Takayama, Phys. Rev. B66, 064431 (2002).

[6] L.F. Cugliandolo, D.R. Grempel, J. Kurchan and E. Vincent, Europhys. Lett. 48, 699 (1999).

[7] D. Hérisson and M. Ocio, Phys. Rev. Lett. 88, 257202 (2002); to appear in Physica C (LT23 Proceedings).

[8] A. Barrat, Phys. Rev. E57, 3629 (1998).

[9] G. Parisi, F. Ricci-Tersenghi and J.J. Ruiz-Lorenzo, Euro. Phys. J. B11, 317 (1999).

[10] E. Marinari, G. Parisi, F. Ricci-Tersenghi and J.J. RuizLorenzo, J. Phys. A33, 2373 (2000).

[11] S. Franz and H. Rieger, J. Stat. Phys. 79, 749 (1995).

[12] H. Kawamura, Phys. Rev. Lett. 80, 5421 (1998).

[13] K. Hukushima and H. Kawamura, Phys. Rev. E61, R1008 (2000), and unpublished.

[14] H. Kawamura and D. Imagawa, Phys. Rev. Lett. 87, 207203 (2001); D. Imagawa and H. Kawamura, J. Phys. Soc. Jpn. 71, 127 (2002).

[15] D. Petit, L. Fruchter and I.A. Campbell, Phys. Rev. Lett. 83, 5130 (1999); 88, 207206 (2002).

[16] V. Dupuis, E. Vincent, J.-P. Bouchaud, J. Hammann, A. Ito and H. Aruga Katori, Phys. Rev. B64, 174204 (2001).

[17] P.E. Jönsson, H. Yoshino, P. Nordblad, H. Aruga Katori and A. Ito, Phys. Rev. Lett. 88, 257204 (2002).

[18] K. Hukushima and H. Kawamura, unpublished.

[19] L. Berthier and J.-P. Bouchaud, Phys. Rev. B66, 054404 (2002).

[20] G.F. Rodriguez, G.G. Kennig and R. Orbach, condmat/0212259.

[21] H. Kawamura, Phys. Rev. Lett. 68, 3785 (1992); Int. J. Mod. Physics C7, 345 (1996). 